\title{
Gambling Addiction in Asia: Need for a Medical Perspective?
}

\author{
David Chim \\ Department of Community Medicine \& School of Public Health, Department of \\ Family Medicine \& Primary Care, The University of Hong Kong, Hong Kong \\ * Corresponding author; email: dchim@hku.hk
}

\begin{abstract}
With the rise of legalized gambling in Macau, Singapore, and other Asian countries, pathological gambling should be more thoroughly discussed by clinical and research professionals in the Asia-Pacific region. Holistic strategies for proper diagnosis, treatment referrals and prevention need to be planned in anticipation of further pro-gambling legislation. Evidence for gambling addiction as a medical condition has been gathered in the West, and studying this social issue via a medical perspective in the East may be useful in the future. Counselors and other professionals working at the frontlines may consider incorporating the medical perspective into their evaluations of pathological gamblers, and collaborating with physicians to provide medical management when appropriate. Such a biopsychosocial approach to pathological gambling may be fruitful in terms of better treatment availability, more holistic care, and decreased stigma for pathological gamblers. Training opportunities for counselors and other professionals should be increased in the Asia-Pacific region for better understanding of gambling addiction as a bio-psychosocial disease.
\end{abstract}

Key words Asia-Pacific - Legalized Gambling ' Medical Disease · Pathological Gambling

\section{Introduction}

With the success of the gambling industry in Macau and now Singapore, gambling in Asia is likely to grow prodigiously in the future. One may speculate that the governing bodies of other countries such as Taiwan, South Korea, and Japan may soon pass legislation in order to emulate 
this success, considering the revenue that can be gained by taxing major legalized gaming establishments.

While Asian social workers and counselors are generally aware of the possibility of increased gambling issues, the perspective of pathological gambling as a chronic medical disease, characterized by abnormal neurobiology, is rarely discussed in their respective communities. Such a timely academic discussion may better prepare clinical professionals (e.g. counselors, psychologists, and physicians) to help the minority of gamblers who develop such an illness. However, a discussion about gambling behavior as an addiction cannot be complete without a review of how it is similar to substance addiction, in terms of diagnostic clinical features, as well as in plausible neurological pathways and neurochemical pathology.

With recent advances in neuroimaging and genomics, substantial evidence is emerging that substance addiction (alcohol, nicotine and illicit drugs) is not merely a complex psychosocial problem but also a chronic medical disease of the brain (Brody et al., 2009; London et al., 2004; Shaffer et al., 2004). Behavioral addictions, such as pathological gambling, are harder to justify as a legitimate medical disease. Hence, we must review previous research on substance abuse and dependence to illustrate how gambling addiction may reasonably be considered a medical disease as well as a psychosocial problem.

Before exploring similarities between gambling addiction and other addictions, it is helpful to review the definitions used by psychiatrists to diagnose substance dependence and abuse. According to the DSM-IV (American Psychiatric Association, 1994), Substance Dependence (to alcohol, nicotine and illicit drugs) can be diagnosed when three or more of the following occurs in a 12-month period: (1) tolerance (increased amounts to reach desired effect, or diminished effect with the same amount of drug); (2) withdrawal; (3) using more in amount or time period than originally intended; (4) persistent desire to cut down use (but unsuccessful); (5) great deal of time spend to acquire and use the substance; (6) important social, occupational, or recreational activities are given up for substance use; and (7) continued use despite negative consequences (American Psychiatric Association, 2000). A less severe DSM-IV diagnosis of Substance Abuse is diagnosed on manifesting one or more of the following in a 12-month period: (1) recurrent substance use resulting in failure in work, school, or home; (2) recurrent substance use in physically hazardous situations; (3) recurrent use resulting in legal problems; and (4) continued use despite interpersonal problems (American Psychiatric Association, 1994). When evaluating pathological gamblers, such diagnostic criteria for substance abuse and dependence are useful to keep in mind.

Currently, pathological gambling is a distinct DSM-IV diagnosis from substance abuse or dependence. It is defined as persistent and recurrent maladaptive gambling behavior as indicated by at least five of the following: (1) preoccupation with gambling; (2) needing to gamble with increasing amounts of money in order to achieve the desired excitement; (3) repeated unsuccessful efforts to control, cut back, or stop gambling; (4) restlessness or irritability when attempting to cut down or stop gambling; (5) gambling as a way of escaping from problems or of relieving 
a dysphoric mood (e.g. anxiety, depression); (6) after losing money gambling, often returns another day to get even; (7) lies to family members, therapists, or others to conceal the extent of involvement with gambling; (8) has committed illegal acts such as forgery, fraud, theft, or embezzlement to finance gambling; (9) has jeopardized or lost a significant relationship, job, or educational or career opportunity because of gambling; and (10) relies on others to provide money to relieve a desperate financial situation caused by gambling (American Psychiatric Association, 1994). Addiction professionals should be vigilant for any change of definition in the upcoming DSM-V, especially for further overlap of clinical features between substance dependence, substance abuse, and pathological gambling.

\section{Evidence of Overlap: Substance and Gambling Addictions}

It is not uncommon for clinical professionals to see individuals with substance use disorders along with other psychiatric co-morbidities, such as pathological gambling. In one study, $28 \%$ of individuals identified as pathological gamblers were concurrently identified as having alcohol dependence, compared with only $1.2 \%$ of the non-pathological gamblers group (Welte et al., 2001). Such an observation warrants further studies as to whether or not certain subgroups of those with substance abuse are more vulnerable to developing pathological gambling, and vice versa. Further, the free-of-charge alcohol and inexpensive cigarettes available in gambling establishments may be harmful for these particular subgroups. It is biologically plausible that alcohol, nicotine and illicit drugs may share neurological pathways with gambling which positively or negatively reinforce such addictions.

Let us review similarities seen between gamblers and drug users, such as in the development of tolerance. Tolerance in gambling may be represented by the increase in amounts of money being wagered over time; patients sometimes report larger and larger bets are needed to experience optimal levels of euphoria (an increased "hedonic tone" in the medical literature). The typical drug addict needs to use increasing amounts of his/her drug of choice to experience the same level of euphoria or "high". Both pathological gamblers and drug addicts may have abnormal neurological brain neurochemistry and pathway patterns for the sensation of reward or pleasure; in short, the neurological pathway of positive reinforcement may have developed into a pathological dopaminergic condition, where only an abnormally high amount of pleasurable activity (taking large amounts of a drug or drugs, gambling huge wagers, etc.) can lead to the desired euphoric sensation.

As with alcoholics and drug addicts, pathological gamblers continue engagement in harmful behavior (taking drugs and gambling, respectively) despite experiencing negative social and occupational ramifications. Like those with substance dependence, pathological gamblers tend to have intensely troubled family relationships, severe financial debts, and often suffer bankruptcy (Lorenz \& Yathe, 1986; National Research Council, 1999). While the similarities discussed suggest that pathological gambling may meet some of the psychiatric criteria for "abuse" (described previously), there is still the need to consider possible evidence 
of physiological consequences that a medical condition of the brain should encompass.

The neurophysiological aspect we seek may be withdrawal syndrome which is a well-known consequence of drug addiction. Withdrawal syndrome is characterized by irritability, psychomotor agitation, decreased concentration, and psychosomatic complaints. Up to $40 \%$ of pathological gamblers suffer from withdrawal syndrome when they stop gambling (Wray \& Dickerson, 1981). Such an observation makes it biologically plausible that both drug addicts and pathological gamblers share similar neurological abnormalities leading to the negative reinforcement of their behavior. In other words, it is possible that their brains share a similar pathology where major unpleasant sensations occur when they stop (or cut down) their addictive behavior. Hence, in order to avoid unpleasant feelings (as opposed to seeking pleasant sensations), these patients continue their addictive behavior.

\section{Previous Neuroimaging Research: Insights to the Abnormal Addicted Brain}

Scholars of problem and pathological gambling may find neuroimaging studies of stimulant-dependent addicts useful for insights into the possible biological aspects of gambling addiction. For example, methamphetamine addicts who abstain from drug use report high levels of depression and anxiety which are accurately correlated with their respective positron emission tomography brain scans (London et al., 2004). During abstinence, the subjects who reported higher levels of depression had higher activity in the amygdala, the brain region responsible for processing of emotions. Subjects who reported higher levels of anxiety had higher amygdala activity and concurrent lower activity in the orbitofrontal cortex, the area of the brain responsible for normal executive functioning. Such measurable evidence of the neurological functioning associated with negative reinforcement in drug addicts warrants further comparable investigation in Asia in pathological gamblers who exhibit withdrawal syndrome.

Positive reinforcement in drug users can be measured in neuroimaging studies of the neurotransmitter dopamine, which is responsible for the sensation of pleasure. The brain process involved is the activation of dopaminergic neurons from the ventral tegmental area to the nucleus accumbens. One study demonstrated that smoking tobacco (also a stimulant) causes more dopamine to be released specifically into the nucleus accumbens (Brody et al., 2009). The study used C-11 Raclopride, a dopamine receptor antagonist that competes with endogenous dopamine for binding to D2 and D3 receptors (dopamine binds to D1, D2, and D3 receptors in the nucleus accumbens). Before smoking, subjects had high binding by C-11 Raclopride in the nucleus accumbens. After smoking, the nicotine from tobacco activated their dopaminergic pathway of positive reinforcement, which was shown by less binding of C-11 Raclopride. This is because nicotine causes endogenous dopamine to be released, and hence C-11 Raclopride had more competition for receptor sites. In the future, neuroimaging studies in Asia may be useful for measuring the euphoria 
(i.e. dopamine receptor occupancy) occurring as a result of gambling cues in certain subgroups of problem or pathological gamblers.

\section{Discussion}

Besides looking at advances in measuring abnormal activity in the brain, noting the overlap of psychological core features of pathological gamblers may be helpful for clinical professionals in frontline treatment services. With the possibility of pathological gambling as a medical condition that involves specific brain pathways, more comprehensive strategies must be explored to deal with this group of vulnerable patients. A holistic assessment of pathological gamblers and their concurrent psychopathological features should be completed at the beginning of every initial client session. This would allow for the stratification of subgroups who may respond to different medical and / or psychosocial treatments. More importantly, taking this medical perspective may aid counselors and social workers to properly refer such clients to medical professionals. Likewise, medical professionals will also need to refer clients to professionals who can provide psychosocial treatment. With the likely rise of gambling establishments in Asia, it may be prudent to create collaborations between physicians, psychologists, social workers, counselors and other professionals to provide the best possible intervention for future pathological gamblers in their respective Asian communities.

As with patients with obsessive-compulsive disorder (OCD), pathological gamblers may have unpleasant feelings (and perhaps the neuro-physiological activation described previously ) which lead to an intense desire to perform a specific behavior (Cartwright et al., 1998), such as betting money in a casino or compulsively buying stocks on the internet, to decrease the unpleasant feelings. It would not be surprising if these unpleasant feelings are mainly due to depression and anxiety, with corresponding increased activity in the amygdala and decreased activity in the orbitofrontal cortex. Both patients with OCD and pathological gamblers have a reduced capacity to resist thoughts of performing specific acts (such as washing one's hands or wagering money); one can hypothesize that neurological mechanisms to stop the pursuit of a pleasurable but harmful activity may have been compromised as part of their chronic brain disease.

Further, pathological gamblers are similar in some respects to another group of medical patients: people suffering from Bipolar Disorder. These two groups are broadly similar in the sense that they continue harmful but pleasurable activity, and often act without forethought-which may represent a decrease in orbitofrontal activity. Both groups can be pleasure-seeking while also having impaired judgment due to an unrealistic assessment of their abilities (Sood et al., 2003). For problem or pathological gamblers, the unrealistic assessment of their abilities would most likely relate to their perceived "luck". Clients often have an irrational belief that their odds of winning are higher than others. Often such unrealistic beliefs are present until the client reaches a stage of ambivalence.

With better understanding of the biological and potential medical aspects of pathological gambling, multidisciplinary teams including addiction medicine 
physicians may be better suited to tackling such a bio-psychosocial problem at the individual and societal level. A neurobiological perspective may help treatment professionals and their clients become more aware of the option of medication treatments, as well as any advances in combined psychosocial treatment and medical management interventions. If medical management for pathological gambling is not available in their respective networks, such a perspective may motivate professionals to seek further training, or participate in research for medical interventions as a regional node. Another potential benefit of a medical perspective would be a reduction in stigma for clients, because pathological gambling would be more accepted as a legitimate medical condition. Culturally, when compared to the West, pathological gambling in Asia may be viewed more as a weakness in character rather than a disease of the brain, which may create considerable shame for those who try to seek help.

Lastly, the integration of the medical perspective allows for better identification and more holistic treatment of pathological gambling subgroups, such as those with co-occurring substance abuse or other psychiatric disorders. A bio-psychosocial system approach which expedites proper diagnosis and treatment of pathological gambling, substance abuse/dependence, and/or other psychiatric illnesses is the ideal infrastructure for gambling counselors and other addiction professionals in Asia to collaborate towards.

\section{References}

American Psychiatric Association. (1994). The diagnostic and statistical manual of mental disorders (4th Edition). Washington, DC: American Psychiatric Association.

Brody, A. L., Mandelkern, M. A., Olmstead, R. E., Allen-Martinez, Z., Scheibal D., Abrams , A. L., ... London, E. D. (2009). Ventral striatal dopamine release in response to smoking a regular vs a denicotinized cigarette. Neuropsychopharmacology, 34, 282-289.

Cartwright, C., DeCaria, C., Hollander, E. (1998). Pathological gambling: a clinical review. Practical Psychiatry and Behavioral Health, 4, 277-286.

London, E. D., Simon, S. L., Berman, S. M., Mandelkern, M. A., Lichtman, A. M., Bramen, J. ... Ling, W. (2004). Mood disturbances and regional cerebral metabolic abnormalities in recently abstinent methamphetamine abusers. Archives of General Psychiatry, 61, 73-84.

Lorenz, V. C., Yathe, R. A. (1986). Pathological gambling: Psychosomatic, emotional, and marital difficulties as reported by the gambler. Journal of Gambling Behavior, 2, 40-49.

National Research Council. (1999). Pathological gambling: A critical review. Washington, DC.

Shaffer, H.J., LaPlante, D.A., LaBrie, R.A., Kidman, R.C., Donato, A.N., Stanton, M.V. (2004). Toward a syndrome model of addiction: multiple expressions, common etiology. Harvard Review of Psychiatry, 12(6), 367-74.

Sood, E. D., Pallanti, S., Hollander, E. (2003). Diagnosis and treat ment of pathological gambling. Current Psychiatry Report, 5, 9-15.

Welte, J., Barnes, G., Wieczorek, W., Tidwell, M. C., Parker, J. (2001). Alcohol and gambling pathology among U.S. adults: Prevalence, demographic patterns and comorbidity. Journal of Studies on Alcohol, 62, 706-712. 
David Chim

Wray, I., Dickerson, M. G. (1981). Cessation of high frequency gambling and "withdrawal" symptoms. British Journal of Addiction, 76, 401-405. 